\title{
Evaluation of the Relationship between Incisal Edge Position of Maxillary Central Incisors and Incisive Papilla in Patients Referred to Prosthodontics Department of Tabriz Dental Faculty
}

Sepideh Bohluli ${ }^{1}$,Alireza Pour Nasrollah ${ }^{2}$,Hosein Eslami ${ }^{1}$,Vahid Fakhrzadeh ${ }^{2}$,Sahar Khadem Neghad ${ }^{1}$, Ramin Negahdari ${ }^{*}$, Seyyed Mahdi Vahid Pakdel ${ }^{2}$

1. Department of Oral Medicine, Faculty of Dentistry, Tabriz University of Medical Sciences, Tabriz, Iran

2. Department of Prosthodontics, Faculty of Dentistry, Tabriz University of Medical Sciences, Tabriz, Iran

\begin{abstract}
Background and Aims: Teeth position is an important component in a facial esthetic. Distance between incisive papilla and incisal edge of the maxillary centrals is a stable landmark in reconstruction anterior teeth of maxilla. Therefore in this study, we tried to establish this index in patients who recourse to the fixed prosthodontics dentistry faculty of Tabriz. Materials and Methods: The initial samples consisted of 59 patients referred to prosthodontics department of Tabriz dental faculty and took upper jaw impressions with irreversible hydrocolloid. Then, records poured by moldana stone until 15 minutes and needed anatomical spots were highlighted. The horizontal distance from anterior part on incisal edge of the maxillary centrals to anterior border, center and posterior border of incisive papillae were measured and statistically inspected. Results: The mean distance between the most anterior point of the incisal edge of the maxillary central incisors and the middle point, anterior border and posterior border of the incisive papilla was $8.54,7.12,10.28 \mathrm{~mm}$ with standard deviations of $0.83,0.75,1.002 \mathrm{~mm}$.

Conclusions: The usefulness of the incisive papilla as a reference point is limited because of the migration and change of shape it has been shown to undergo. However, it may serve some purpose because it is an easy measurement to make. Although the clinical findings may not always be true, but in many cases there is a close relationship.
\end{abstract}

Key words: Teeth; incisive papilla; maxilla; occlusal plane;

\section{Introduction}

Tooth alignment is one of the important factors affecting facial esthetic. Reconstruction of teeth during prosthesis treatment based on esthetic, phonetics, patient comfort and psychological factors, has notable effect on social relations of the patients (5).

Edentulism alters human's face, especially lower one third, and this is dentist's duty to restore the natural profile of the smile backed by his/ her knowledge and skill (1).

\section{Corresponding author:}

Ramin Negahdari

Department of Prosthodontics, Faculty of Dentistry, Tabriz University of Medical Sciences, Tabriz, Iran E-mail: Ramin_n_dds@yahoo.com

Receive date: 2016-05-25| Accept date: 2016-06-29| Publish date: 2016-07-28

DOI: 10.7575/aiac.abcmed.16.04.03.05 
To preserve the facial appearance in edentulous patients beside the correct selection of artificial teeth according to their color, shape and size, teeth must be place in the correct position.

Without doubt, well-positioned and nice teeth have undeniable influence on appearance and central teeth have prominent role in comparison to others. To achieve a natural appearance, it is essential that anterior teeth have to be placed in their original location during fabrication of denture because in such position, the soft tissue benefits the teeth support which brings about a younger appearance. Determination of the correct position of incisor teeth is one of the important considerations in fabrication of complete denture or implant based prosthesis (7).

The anterior part of maxilla undergoes extensive changes. According to previous studies, after edentulism alveolar process atrophies with different paces. We can benefit current denture, photos before edentulism, diagnostic casts and the radiographies before edentulism to determine the teeth position. Facial structure of edentulous patient can indirectly indicates the original position of natural teeth. Determination of the appropriate position of artificial teeth can be achieved by biometric measurements (3).

To place the anterior artificial teeth in their correct mesio-distal position, generally facial midline is used. Sometimes we have to consider other anatomic landmarks like labial frenum and incisive papilla or palatal suture to determine the midline in people who suffers from asymmetry (7). Incisive papilla which is placed on palatal midline, between central teeth have substantial role in determining the position of central teeth as a land mark. This index is an adequate landmark to place central teeth in their proper position in complete denture, partial denture and implant (6). Lynn, after investigating Anatomical layers of oral cavity and face, concluded that we can use incisive papilla as teeth placement guidance (7). Lombardi studied the relation between central teeth and incisive papilla and finally concluded that it can be used as a reliable landmark for arranging teeth because of its stable relation with central teeth (8).

Latta carried out a study on 100 patients and averagely in 78 patients, the midline of central teeth is $1 \mathrm{~mm}$ away from nasopalatine papilla midline. The average distance of labial frenum from midline is about $0.93 \mathrm{~mm}(0-5.5 \mathrm{~mm})$. In this study, age and gender cause no significant difference (1). In 1984 Harpper noted that the incisal edge of central teeth is horizontally 5-8 $\mathrm{mm}$ away from the middle of incisive papilla (3), while Hickeg and Martone suggested 8-10 $\mathrm{mm}$ (9). Erlich and Gazit found out that the labial surface of central teeth is averagely 12 to 13 away from incisive papilla (8). According to Ortman et al, the distance between labial surface central teeth and the posterior boarder of incisive papilla is $12.5 \mathrm{~mm}$ (7).

\section{Materials and Methods:}

Patients, who were younger than 18, lost their one or two central teeth, suffered from severe central teeth attrition or severe crowding, wore fixed prosthesis in central teeth location, were put out of the study.

59 patients, who meat entry criteria, were selected out of visitors of the Tabriz Dentistry Faculty. A maxillary alginate impression (Aleginoplast- Bayer dental- LeverkusenGermany) was taken under due consideration. Mixing of the impression material was done following the factory instruction. During the preparation of impression we paid high attention to avoid displacing the labial frenum position. The impressions were casted maximally within 15 minutes with moldana plaster. 45 minutes after ultimate setting of plaster, the impressions and casts were separated. 
All castes were trimmed according to orthodontics standard angles. Castes were put in slurry water for 15 minutes before trimming and after scratching with soft brush, slurry water washed away carefully so details were preserved. When the casts were laid on a horizontal surface, the occlusal plan had to be parallel with horizontal plane.

Middle, anterior and posterior borders of incisive papilla were marked with pencil and the distance between these three points and most anterior part of incisal region of central teeth were measured with diametric collis. To be sure that the measurement was done horizontally, casts were trimmed according to orthodontics trimming principles, so that the occlusal plane was parallel with horizontal plan. On the other hand collis was modified in a way that makes it possible to hold it horizontally during measurement. All measurements were done by the same operator so that mistake caused by several observes was ruled out.

In some cases because of integration of papilla with peripheral connective tissue, it was impossible to exactly mark borders, so these cases were put out of study.

\section{Results:}

To analyze the horizontal distance between incisal edge and middle, anterior and posterior borders of incisive papilla in studied population, we used Average test and central indexes. Based on the results, we can see that the horizontal distance between incisal edge of central teeth and incisive papilla in studied population is averagely equal with $8.54 \mathrm{~mm}$ also the following distance when we use the anterior border of incisive papilla averagely equals with $7.120 .75 \mathrm{~mm}$ and for posterior border it is equal with $10.281 .002 \mathrm{~mm}$.

\section{Discussion:}

Arranging the teeth in way that they seem natural is one of the artistic skills of a dentist.
Butcher et al stated that the statue and appearance of lips and lower part of the face are the best guidance for arranging teeth while other factors are coming next. Absolute accuracy and correctness in arranging the teeth

in anterior region doesn't seem rational. Changes which are undergone by muscles and alveolar bone after edentulism bring about new demands.

Advantages of using incisive papilla as a reference point are limited since these structures migrate gradually and undergo some changes. Any way it satisfies some demands because of easy measurement (8).

The measurement of the distance between the labio-incisal one third of central teeth and anterior, posterior border and middle boarder of incisive papilla can help us determine the location of anterior teeth. Incisal papilla is made of fibrous connective tissue covering the bony orifice, out of which the nasopalatine nerves and vessels exit.

This area mustn't be pressed or displaced during taking impression. Pressure produced by denture on the area causes pain or parasthesia (7).

Mean value of the distance between the incisal edge of the central teeth and the middle point of papilla is $8.54 \mathrm{~mm}$ with the standard deviation of $0.83 \mathrm{~mm}$. our results were in 8 to $10 \mathrm{~mm}$ interval and comparable with Samiris' ones $(8.50 \mathrm{~mm})$ and Mavroskoafis and Ritchie's $(10.2 \mathrm{~mm})$. Anyway, the convergence in the reference point of the incisal edge is not ignorable (9).

In spite of the other studies which solely measured the distance of the most anterior part of the incisal edge from one specific point, in our study the measurement was taken, using three points marked on incisive papilla. The following difference makes our study more comprehensive. 
Also in the current study by defining more exit criteria we were up to reach more definite results. In the previous studies the exit criteria were limited to having fix prosthesis in anterior region and loss of one or two central teeth.

Considering the incisal papilla as guidance for arranging teeth bases on a hypothesis that says the incisive papilla is a fixed landmark. Although Haper believes that the incisal papilla doesn't migrate after 7 years of extraction, he didn't provide enough proof.

Watt and Likemen studied the recession patter of alveolar ridge for 2.5 years in 25 patients and found that the incisive papilla averagely migrates $1.6 \mathrm{~mm}$ forward (0.76 to $2.77 \mathrm{~mm}$ ) and $2.3 \mathrm{~mm}$ upward (1.3 to $4 \mathrm{~mm}$ ). During the recession, the relation of incisive papilla and incisive foramen alters. The upward and forward changes in incisive papilla have to be considered if it will be used as guidance for the arranging of anterior teeth (9).

In this study the mean distance between incisors and papilla is $8.54 \mathrm{~mm}$ which is in 8 to $10 \mathrm{~mm}$ interval. Anyway it is not rational that a dentist applies these values exactly for every patient. Individual differences have to be considered. The $8.54 \mathrm{~mm}$ with $0.83 \mathrm{~mm}$ standard deviation shows that the $68 \%$ of population is somewhere between 8.06 to $10.28 \mathrm{~mm}$. In other words $30 \%$ of population is out of 8 to $10 \mathrm{~mm}$ interval. Nowadays it is recommended not to place the teeth exactly in their former natural position because of alternation in personality traits, jaws relation and lips because of edentulism (9).

Instead of insisting on a defined pattern, dentist should use a combination of biometric guidance, phonetic tests and measurement of the denture space to reconstruct the relation between oral tissues and natural teeth properly. A technician because of the lack of knowledge about clinical land marks is obliged to use anatomical land marks, so arranged teeth placed almost near to their ultimate position, and it is dentist's duty to decide about the optimum position of teeth according to mentioned considerations.

This method helps reducing the chair side time. Dentist, after receiving the occlusion rim, adjusts it according to personal demands of patient (4).

The resulted statistical data can be an ideal point to start to fabricate occlusion rim. Also this data can be a reliable guidance for clinical adjustment of maxillary incisor teeth and positioning of these teeth on the base of esthetic and phonetic in try-in session.

\section{Conclusions:}

The usefulness of the incisive papilla as a reference point is limited because of the migration and change of shape it has been shown to undergo. However, it may serve some purpose because it is an easy measurement to make. Although the clinical findings may not always be true, but in many cases there is a close 


\section{References}

1. Ghrachahi J, Mahdavian SJ, Asadzadeh Aghdaei N, Rahbar MA. An investigation on the facial midline distance to some anatomic landmarks of the jaws. Journal of Dentistry 2008; 18: 41-51.

2. Mosharraf R, Abtahi D. Porrabani M . An investigation on the facial midline distance to some anatomic landmarks of the jaws among people with natural dentition. JDM 2003; 16:31-38.

3. Seok Park Y, Cumhur A, Lee SP, Paik KS. The three dimensional relationship on a virtual model between the maxillary anterior teeth and incisive papilla. J Prosthet Dent. 2007;98:312-8.

4. Ortman HR, Tsao DH. Relationship of the incisive papilla to the maxillary central incisors. The Journal of Prosthetic Dentistry 1979;42:492-496.

5. Ustun Guldag M, Sentut F, Buyukkaplan S. Investigation of vertical distance between incisive papilla and incisal edge of maxillary central incisors. European Journal of Dentistry 2008;2:161-166.

6. Boucher CO. Complete denture impresion based upon the anatomy of the mouth, J.A.D.A. 31:1174-1181, 1944.

7. Harper RN. The incisive papilla. J Dent Res 1948; 27:661-8.

8. Grave $\mathrm{AMH}$, Becher PJ. Evaluation of incisive papilla as a guide to anterior tooth position. J Prosthet Dent. 1987;57:712-4.

9. Lau GCK, Cumhur A, Clark RFK. The relationship of the incisive papilla to the maxillary central incisors and canine teeth in southern chinese. J Prosthet Dent. 1993;70:86-93. 